Pacific

Journal of

Mathematics

\title{
ALMOST FACTORIALITY OF INTEGRAL DOMAINS AND KRULL-LIKE DOMAINS
}

Gyu Whan Chang, Hwankoo Kim and Jung Wook Lim 


\title{
ALMOST FACTORIALITY OF INTEGRAL DOMAINS AND KRULL-LIKE DOMAINS
}

\author{
Gyu Whan Chang, Hwankoo Kim and Jung Wook Lim
}

\begin{abstract}
Let $D$ be an integral domain, $\bar{D}$ be the integral closure of $D$, and $\Gamma$ be a numerical semigroup with $\Gamma \subsetneq \mathbb{N}_{0}$. Let $t$ be the so-called $t$-operation on $D$. We will say that $D$ is an AK-domain (resp., AUF-domain) if for each nonzero ideal $\left(\left\{a_{\alpha}\right\}\right)$ of $D$, there exists a positive integer $n=n\left(\left\{a_{\alpha}\right\}\right)$ such that $\left(\left\{a_{\alpha}^{n}\right\}\right)_{t}$ is $t$-invertible (resp., principal). In this paper, we study several properties of AK-domains and AUF-domains. Among other things, we show that if $D \subseteq \bar{D}$ is a bounded root extension, then $D$ is an AK-domain (resp., AUFdomain) if and only if $\bar{D}$ is a Krull domain (resp., Krull domain with torsion $t$-class group) and $D$ is $t$-linked under $\bar{D}$. We also prove that if $D$ is a Krull domain (resp., UFD) with $\operatorname{char}(D) \neq 0$, then the (numerical) semigroup ring $D[\Gamma]$ is a nonintegrally closed AK-domain (resp., AUF-domain).
\end{abstract}

\section{Introduction}

Throughout this paper, $D$ is an integral domain with quotient field $K, \bar{D}$ denotes the integral closure of $D$ in $K, X$ is an indeterminate over $D, D[X]$ is the polynomial ring over $D, \mathbb{N}_{0}$ (resp., $\mathbb{Z}$ ) is the set of nonnegative integers (resp., integers), $\Gamma$ is a numerical semigroup with $\Gamma \subsetneq \mathbb{N}_{0}$ and $D[\Gamma]$ means the numerical semigroup ring of $\Gamma$ over $D$.

We say that $D$ is a $G C D$-domain if $a D \cap b D$ is principal for all $0 \neq a, b \in D$. In [Zafrullah 1985], Zafrullah introduced the notion of an almost GCD-domain (AGCD-domain). He called $D$ an AGCD-domain if for each $0 \neq a, b \in D$, there exists an integer $n=n(a, b) \geq 1$ such that $a^{n} D \cap b^{n} D$ is principal. After Zafrullah's paper [1985], several types of almost divisibility of integral domains have been studied, for example, AB-domains, AP-domains, APvMDs, API-domains and ADdomains (see Section 1). Recall from [Kang 1989a] that $D$ is a Krull domain (resp., UFD) if and only if for every nonzero ideal $I$ of $D, I_{t}$ is $t$-invertible (resp.,

The second author was supported by the Basic Science Research Program through the National Research Foundation of Korea (NRF) funded by the Ministry of Education, Science and Technology (2010-0011996). The third author was supported by the Brain Korea 21 Project Team to Nurture the Next Generation of First-class Mathematical Scientists by the Korean Government.

MSC2010: primary 13F05; secondary 13A15, 13B25, $13 \mathrm{G} 05$.

Keywords: AK-domain, AUF-domain, numerical semigroup, bounded root extension. 
principal). In this paper, we define $D$ to be an $A K$-domain (resp., AUF-domain) if for each nonzero ideal $\left(\left\{a_{\alpha}\right\}\right)$ of $D$, there exists a positive integer $n=n\left(\left\{a_{\alpha}\right\}\right)$ such that $\left(\left\{a_{\alpha}^{n}\right\}\right)_{t}$ is $t$-invertible (resp., principal). (For the sake of convenience, we will use the notation $\left\{a_{\alpha}\right\}$ instead of $\left\{a_{\alpha}\right\}_{\alpha \in \Lambda}$, where $\Lambda$ is an indexed set. Also, for a nonempty subset $\left\{a_{\alpha}\right\}$ of $D \backslash\{0\}$, we mean by $\left(\left\{a_{\alpha}\right\} D\right)$ the ideal of $D$ generated by the set $\left\{a_{\alpha}\right\}$.)

In Section 1, we review multifarious integral domains related to the theory of almost divisibility and some results on them.

We devote Section 2 to the study of AK-domains. Precisely, we show that if $D \subseteq \bar{D}$ is a bounded root extension, then $D$ is an AK-domain if and only if $\bar{D}$ is a Krull domain and $D$ is $t$-linked under $\bar{D}$, if and only if (i) $t-\operatorname{dim}(D)=1$, (ii) $D_{P}$ is an API-domain for each $P \in t-\operatorname{Max}(D)$ and (iii) $D=\bigcap_{P \in t-\operatorname{Max}(D)} D_{P}$ and this intersection has finite character. We prove that if $D$ is a Krull domain, then $D[\Gamma]$ is an AK-domain if and only if $\operatorname{char}(D) \neq 0$. This result can be used to construct a simple example of nonintegrally closed AK-domains. We also prove that the (numerical) semigroup ring $D[\Gamma]$ is an AK-domain if and only if $D[X]$ is an AK-domain and $\operatorname{char}(D) \neq 0$.

In Section 3, we introduce the notions of an AUF-domain and an almost $\pi$ domain. We show that $D$ is an AUF-domain (resp., almost $\pi$-domain) if and only if $D$ is an AK-domain and $C l(D)$ is torsion (resp., $G(D)$ is torsion). We prove that $D[\Gamma]$ is an AUF-domain if and only if $D[X]$ is an $\operatorname{AUF-domain~and~} \operatorname{char}(D) \neq 0$. Also, we show that if $D$ is a UFD (resp., $\pi$-domain), then $D[\Gamma]$ is an AUF-domain (resp., almost $\pi$-domain) if and only if $\operatorname{char}(D) \neq 0$. Finally, we give an example of an AUF-domain that is neither integrally closed nor an API-domain.

Now, we review some definitions and notation. Let $\mathbf{F}(D)$ be the set of nonzero fractional ideals of $D$. For an $I \in \mathbf{F}(D)$, we denote by $I^{-1}$ the fractional ideal $\{x \in K \mid x I \subseteq D\}$ of $D$. Recall that the $v$-operation on $D$ is the mapping on $\mathbf{F}(D)$ defined by $I \mapsto I_{v}=\left(I^{-1}\right)^{-1}$, and the $t$-operation on $D$ is the mapping on $\mathbf{F}(D)$ defined by $I \mapsto I_{t}=\bigcup\left\{J_{v} \mid J\right.$ is a nonzero finitely generated fractional subideal of $I$ \}. Clearly, if an $I \in \mathbf{F}(D)$ is finitely generated, then $I_{t}=I_{v}$. An $I \in \mathbf{F}(D)$ is called a $t$-ideal (resp., $v$-ideal) if $I_{t}=I$ (resp., $I_{v}=I$ ). An $I \in \mathbf{F}(D)$ is said to be $v$-finite type if $I_{v}=J_{v}$ for some finitely generated ideal $J$ of $D$. A $t$-ideal $M$ of $D$ is called a maximal $t$-ideal if $M$ is maximal among proper integral $t$-ideals of $D$. Let $t$-Max $(D)$ be the set of maximal $t$-ideals of $D$. It is known that $t-\operatorname{Max}(D) \neq \varnothing$ if $D$ is not a field; a prime ideal minimal over a $t$-ideal is a $t$-ideal; a maximal $t$-ideal is a prime ideal; and each proper integral $t$-ideal is contained in a maximal $t$-ideal. We say that $D$ has $t$-dimension one, denoted by $t-\operatorname{dim}(D)=1$, if each maximal $t$-ideal is of height one. An $I \in \mathbf{F}(D)$ is said to be $t$-invertible if $\left(I I^{-1}\right)_{t}=D$; equivalently, $I I^{-1} \nsubseteq M$ for all $M \in t$-Max $(D)$. It was shown that an $I \in \mathbf{F}(D)$ is $t$-invertible if and only if $I_{v}=J_{v}$ for some finitely generated fractional ideal $J$ of 
$D$ and $I D_{M}$ is principal for each $M \in t-\operatorname{Max}(D)$ [Kang 1989b, Corollary 2.7]. We say that $D$ is a Prüfer $v$-multiplication domain $(\mathrm{P} v \mathrm{MD})$ if each nonzero finitely generated ideal of $D$ is $t$-invertible. A nonzero prime ideal $Q$ of $D[X]$ is called an upper to zero in $D[X]$ if $Q \cap D=(0)$. As in [Houston and Zafrullah 1989], we say that $D$ is a $U M T$-domain if every upper to zero in $D[X]$ is $t$-invertible. It is known that $D$ is an integrally closed UMT-domain if and only if $D$ is a $\mathrm{P} v \mathrm{MD}$ [Houston and Zafrullah 1989, Proposition 3.2]

Let $T(D)$ be the abelian group of $t$-invertible fractional $t$-ideals of $D$ under the $t$-multiplication $I * J=(I J)_{t}$ and $\operatorname{Inv}(D)$ (resp., $\left.\operatorname{Prin}(D)\right)$ be the subgroup of $T(D)$ of invertible (resp., principal) fractional ideals of $D$. Then it is obvious that $\operatorname{Prin}(D) \subseteq \operatorname{Inv}(D) \subseteq T(D)$. The $t$-class group of $D$ is an abelian group $C l(D)=T(D) / \operatorname{Prin}(D)$ and the Picard group $\operatorname{Pic}(D)=\operatorname{Inv}(D) / \operatorname{Prin}(D)$ of $D$ is a subgroup of $C l(D)$. The local $t$-class group $G(D)$ of $D$ is defined by $G(D)=$ $C l(D) / \operatorname{Pic}(D)$.

Let $D \subseteq E$ be an extension of integral domains. Then $E$ is said to be a root extension of $D$ if for each $z \in E, z^{n} \in D$ for some $n \geq 1$. We say that $D \subseteq E$ is a bounded root extension if there exists a fixed positive integer $n$ such that $z^{n} \in D$ for all $z \in E$. The domain $D$ is said to be root closed if for $z \in K, z^{n} \in D$ for some integer $n \geq 1$ implies $z \in D$.

A numerical semigroup is a nonempty subset $\Gamma$ of $\mathbb{N}_{0}$ that is closed under addition, contains 0 and generates $\mathbb{Z}$ as a group. It is known that the set $\mathbb{N}_{0} \backslash \Gamma$ is finite, and $\Gamma$ has a unique numerical system of generators. Also, there always exists the largest nonnegative integer that is not contained in $\Gamma$. Such an integer is called the Frobenius number of $\Gamma$ and is denoted by $F(\Gamma)$. If $\Gamma \subsetneq \mathbb{N}_{0}$, then $D+X^{F(\Gamma)+1} D[X] \subseteq D[\Gamma] \subsetneq D[X]$.

Our general reference for results from multiplicative ideal theory will be [Gilmer 1992]. For any undefined terms, readers are referred to [Kaplansky 1994].

\section{Almost divisibility of integral domains}

In multiplicative ideal theory, one of the important topics during the past few decades was the theory of factorizations in integral domains. Among various kinds of integral domains, many mathematicians have studied Bézout domains, Prüfer domains, principal ideal domains (PID) and Dedekind domains. As for the $t$-operation analogues, they have also investigated GCD-domains, PvMDs, generalized GCD-domains (GGCD-domain), unique factorization domains (UFD), $\pi$-domains and Krull domains.

In [Storch 1967], almost factorial domains were studied as Krull domains with torsion divisor class groups. Motivated by this, Zafrullah first began to study a 
general theory of almost factoriality. To do this, he first defined an almost $G C D$ domain (AGCD-domain) that is an integral domain $D$ in which for each $0 \neq a, b \in D$, there exists an integer $n=n(a, b) \geq 1$ such that $\left(a^{n}, b^{n}\right)_{t}$, equivalently $a^{n} D \cap b^{n} D$, is principal [Zafrullah 1985]. He also showed that $D$ is an integrally closed AGCDdomain if and only if $D$ is a $\mathrm{P} v \mathrm{MD}$ with torsion $t$-class group [Zafrullah 1985, Corollary 3.8 and Theorem 3.9].

Anderson and Zafrullah continued the investigation of AGCD-domains and introduced several closely related domains. They proved that $D$ is an AGCD-domain if and only if $\bar{D}$ is an AGCD-domain [Anderson and Zafrullah 1991, Section 4], $D \subseteq \bar{D}$ is a root extension and $D$ is $t$-linked under $\bar{D}$ [ibid., Theorem 5.9]. (See the remark after Example 2.4 for the definition of " $t$-linked under".) They also defined AB-domains and AP-domains as follows: $D$ is an almost Bézout domain (AB-domain) (resp., almost Prüfer domain (AP-domain)) if for each $0 \neq a, b \in D$, there exists a positive integer $n=n(a, b)$ such that $\left(a^{n}, b^{n}\right)$ is principal (resp., invertible). They proved that $D$ is an AB-domain if and only if $D$ is an AP-domain with torsion $t$-class group [ibid., Lemma 4.4]. They also showed that $D$ is an AP-domain (resp., AB-domain) if and only if $\bar{D}$ is a Prüfer domain (resp., with torsion ( $t$-)class group) and $D \subseteq \bar{D}$ is a root extension [ibid., Corollary 4.8].

Recently, Li introduced the concept of almost Prüfer $v$-multiplication domains. She defined $D$ to be an almost Prüfer $v$-multiplication domain (APvMD) if for each $0 \neq a, b \in D$, there exists a positive integer $n=n(a, b)$ such that $\left(a^{n}, b^{n}\right)_{v}$, equivalently $a^{n} D \cap b^{n} D$, is $t$-invertible [Li 2012, Definition 2.1 and Theorem 2.3]. It was shown that $D$ is an AGCD-domain if and only if $D$ is an $\mathrm{AP} v \mathrm{MD}$ with torsion $t$-class group [Li 2012, Theorem 3.1].

The notion of an almost generalized GCD-domain was first introduced by Anderson and Zafrullah [1991, Section 3], and was also investigated by Lewin [1997]. (Recall that $D$ is an almost generalized GCD-domain (AGGCD-domain) if for each $0 \neq a, b \in D$, there is an integer $n=n(a, b) \geq 1$ such that $\left(a^{n}, b^{n}\right)_{v}$ is invertible.) In [Chang et al. 2012], we studied AGGCD-domains further. We showed that $D$ is an AGGCD-domain if and only if $D$ is an $\mathrm{AP} v \mathrm{MD}$ and $G(D)$ is torsion [Chang et al. 2012, Theorem 2.11]. This result corrects an error in [Lewin 1997, Theorem 5.2], which incorrectly states that $D$ is an integrally closed AGGCD-domain if and only if $D$ is a $\mathrm{P} v \mathrm{MD}$ and $G(D)=0$. (See the review of [Lewin 1997] in Mathematical Reviews database for more details.)

Assume that $D$ is integrally closed. If $D$ is an AB-domain or an AP-domain, then $D$ is a Prüfer domain [Anderson and Zafrullah 1991, Theorem 4.7]. Also, if $D$ is an $\mathrm{AP} v \mathrm{MD}$, an AGCD-domain or an AGGCD-domain, then $D$ is a $\mathrm{P} v \mathrm{MD}$ [Li 2012, Theorem 2.4; Lewin 1997, Theorem 5.2]. As mentioned above, Prüfer domains and $\mathrm{P} v$ MDs have been much studied in the context of factorization theory, and there are many well-known results about them. So, from this point of view, integrally 
closed domains are no longer of interest in the theory of almost factoriality. This is why we need to investigate the almost divisibility of nonintegrally closed domains. One of the requisites to study them is to find some examples of such domains. Among several methods, in [Chang et al. 2012], the authors gave simple examples of nonintegrally closed AP $v$ MDs, AGCD-domains, AGGCD-domains, AP-domains and AB-domains via the $D+X^{n} K[X]$ constructions. In fact, they proved that for an integer $n \geq 2, D+X^{n} K[X]$ is an AP $v$ MD (resp., AGCD-domain, AGGCD-domain, AP-domain, AB-domain) if and only if $D$ is an APvMD (resp., AGCD-domain, AGGCD-domain, AP-domain, AB-domain) and $\operatorname{char}(D) \neq 0$ [Chang et al. 2012, Theorem 2.6 and Corollaries 2.10 and 2.13].

Anderson and Zafrullah [1991] defined an AD-domain (resp., almost principal ideal domain (API-domain)) to be a domain $D$ such that for any nonempty subset $\left\{a_{\alpha}\right\} \subseteq D \backslash\{0\}$ there exists a positive integer $n=n\left(\left\{a_{\alpha}\right\}\right)$ with $\left(\left\{a_{\alpha}^{n}\right\}\right)$ invertible (resp., principal). They showed that $D$ is an API-domain if and only if $D$ is an AD-domain with torsion $t$-class group [Anderson and Zafrullah 1991, Lemma 4.4]. Moreover, if $D \subseteq \bar{D}$ is a bounded root extension, then $D$ is an AD-domain (resp., API-domain) if and only if $\bar{D}$ is a Dedekind domain (resp., $\bar{D}$ is a Dedekind domain with torsion class group) [ibid., Corollary 4.13].

As "almost" versions of Krull domains and UFDs, we will define and investigate AK-domains and AUF-domains in Sections 2 and 3, respectively. We also provide an example of AK-domains (resp., AUF-domains) which is not a Krull domain (resp., UFD).

\section{AK-domains}

Let $D$ be an integral domain with quotient field $K, \bar{D}$ be the integral closure of $D$ in $K, X^{1}(D)$ be the set of height-one prime ideals of $D, \Gamma$ be a numerical semigroup with $\Gamma \subsetneq \mathbb{N}_{0}$, and $D[\Gamma]$ be the (numerical) semigroup ring of $\Gamma$ over $D$.

We say that $D$ is an $A K$-domain if for each nonzero ideal $\left(\left\{a_{\alpha}\right\}\right)$ of $D$, there exists a positive integer $n=n\left(\left\{a_{\alpha}\right\}\right)$ such that $\left(\left\{a_{\alpha}^{n}\right\}\right)$, equivalently, $\left(\left\{a_{\alpha}^{n}\right\}\right)_{t}$ is $t$-invertible. We have avoided using the terminology "almost Krull" for an AK-domain because the term "almost Krull" is already used to mean an integral domain that is locally Krull in the literature [Pirtle 1968]. Clearly, AK-domains are APvMDs; so if $D$ is an AK-domain, then $D$ is a UMT-domain and $D \subseteq \bar{D}$ is a root extension [ $\mathrm{Li}$ 2012, Theorem 3.8]. Also, a Krull domain is an AK-domain, but not vice versa. For example, if $m \equiv 5(\bmod 8)$, then $\mathbb{Z}[\sqrt{m}]$ is a nonintegrally closed API-domain [Anderson and Zafrullah 1991, Theorem 4.17]; so $\mathbb{Z}[\sqrt{m}]$ is an AK-domain that is not a Krull domain. Note that if $D$ is integrally closed, then $\left(\left\{a_{\alpha}^{n}\right\}\right)_{t}=\left(\left(\left\{a_{\alpha}\right\}\right)^{n}\right)_{t}$ for any integer $n \geq 1$ and any nonempty subset $\left\{a_{\alpha}\right\}$ of $D \backslash\{0\}$ [Anderson and Zafrullah 1991, Corollary 6.4]. Thus, an integrally closed AK-domain is a Krull domain. 
Our first result is the AK-domain analogue of the well-known fact that $D$ is a Dedekind domain if and only if $D$ is a Krull domain and each maximal ideal is a $t$-ideal.

Proposition 2.1. $D$ is an $A D$-domain if and only if $D$ is an $A K$-domain and each maximal ideal of $D$ is a $t$-ideal.

Proof. $(\Rightarrow$ ) Since an AD-domain is an AP-domain, by [Chang et al. 2012, Remark before Theorem 2.6], each maximal ideal of $D$ is a $t$-ideal. Clearly, $D$ is an AK-domain.

$(\Leftarrow)$ Let $\left(\left\{a_{\alpha}\right\}\right)$ be a nonzero ideal of $D$. Since $D$ is an AK-domain, there is a positive integer $m=m\left(\left\{a_{\alpha}\right\}\right)$ such that $\left(\left\{a_{\alpha}^{m}\right\}\right)$ is $t$-invertible. So $\left(\left\{a_{\alpha}^{m}\right\}\right)\left(\left\{a_{\alpha}^{m}\right\}\right)^{-1} \nsubseteq P$ for all $P \in t$-Max $(D)$, and since each maximal ideal is a $t$-ideal, $\left(\left\{a_{\alpha}^{m}\right\}\right)\left(\left\{a_{\alpha}^{m}\right\}\right)^{-1} \nsubseteq M$ for all maximal ideals $M$ of $D$. Thus $\left(\left\{a_{\alpha}^{m}\right\}\right)\left(\left\{a_{\alpha}^{m}\right\}\right)^{-1}=D$.

Let $D$ be a Noetherian domain. It is known that $D$ is a UMT-domain if and only if $t-\operatorname{dim}(D)=1$ [Houston and Zafrullah 1989, Theorem 3.7]; so if $D$ is an AK-domain, then $t$-dim $(D)=1$. But, we do not know whether an AK-domain is generally of $t$-dimension one.

We next give a characterization of AK-domains under the " $t$-dimension one" assumption, which turns out to be very similar to that of a Krull domain as described in its definition: $D$ is called a Krull domain if (i) $D=\bigcap_{P \in X^{1}(D)} D_{P}$, (ii) $D_{P}$ is a local PID for each $P \in X^{1}(D)$ and (iii) the intersection $D=\bigcap_{P \in X^{1}(D)} D_{P}$ has finite character, i.e., each nonzero element $d \in D$ is a unit in $D_{P}$ for all but a finite number of $P$ 's in $X^{1}(D)$.

Theorem 2.2. If $t$-dim $(D)=1$, then $D$ is an $A K$-domain if and only if

(1) $D_{P}$ is an API-domain for each $P \in t-\operatorname{Max}(D)$, and

(2) $D=\bigcap_{P \in t-\operatorname{Max}(D)} D_{P}$ and this intersection has finite character.

Proof. $(\Rightarrow)(1)$ Let $\left\{a_{\alpha}\right\}$ be a nonempty subset of $D \backslash\{0\}$. Since $D$ is an AK-domain, there exists a positive integer $n=n\left(\left\{a_{\alpha}\right\}\right)$ such that $\left(\left\{a_{\alpha}^{n}\right\}\right)$ is $t$-invertible. Hence $\left(\left\{a_{\alpha}^{n}\right\}\right) D_{P}$ is principal for each $P \in t-\operatorname{Max}(D)$ [Kang 1989b, Corollary 2.7]. Thus $D_{P}$ is an API-domain.

(2) Note that $D=\bigcap_{P \in t-\operatorname{Max}(D)} D_{P}$ [Kang 1989b, Proposition 2.9]. If $P=$ $\left(\left\{a_{\alpha}\right\}\right) \in t-\operatorname{Max}(D)$, then there is an integer $n=n(P) \geq 1$ such that $\left(\left\{a_{\alpha}^{n}\right\}\right)$ is $t$-invertible, and hence $\left(\left\{a_{\alpha}^{n}\right\}\right)_{t}=\left(a_{1}, \ldots, a_{k}\right)_{t}$ for some $a_{1}, \ldots, a_{k} \in P$. Clearly, $P=\sqrt{\left(a_{1}, \ldots, a_{k}\right)_{t}}$. If $0 \neq d \in D$, then each maximal $t$-ideal of $D$ containing $d$ is minimal over $d D$ because $t-\operatorname{dim}(D)=1$. Thus $D$ has a finite number of maximal $t$-ideals that contain $d$ [Sahandi 2010, Corollary 2.5], since each maximal $t$-ideal is the radical of a finite type $t$-ideal.

$(\Leftarrow)$ Let $\left(\left\{a_{\alpha}\right\}\right)$ be a nonzero ideal of $D$. By $(2)$, the number of maximal $t$-ideals of $D$ containing $\left\{a_{\alpha}\right\}$ is finite, say $P_{1}, \ldots, P_{k}$. Since each $D_{P_{i}}$ is an API-domain 
by (1), there exists a positive integer $m_{i}$ such that $\left(\left\{a_{\alpha}^{m_{i}}\right\}\right) D_{P_{i}}$ is principal for each $i=1, \ldots, k$, say $\left(\left\{a_{\alpha}^{m_{i}}\right\}\right) D_{P_{i}}=\left(a_{i}\right) D_{P_{i}}$ for some $a_{i} \in\left\{a_{\alpha}^{m_{i}}\right\}$. Let $m=m_{1} \cdots m_{k}$ and set $\hat{m}_{i}=m / m_{i}$ for each $i=1, \ldots, k$. Then $\left(\left\{a_{\alpha}^{m}\right\}\right) D_{P_{i}}=\left(a_{i}^{\hat{m}_{i}}\right) D_{P_{i}}$ for all $i=1, \ldots, k$ [Gilmer 1992, Theorem 6.5(c)]; so $\left(\left\{a_{\alpha}^{m}\right\}\right)$ is $t$-locally principal and $\left(\left\{a_{\alpha}^{m}\right\}\right)$ is contained in only a finite number of maximal $t$-ideals of $D$. Choose any nonzero element $a \in\left(\left\{a_{\alpha}^{m}\right\}\right)$. By (2), there exist only finitely many maximal $t$-ideals $M_{1}, \ldots, M_{n}$ of $D$ containing $a$. Let $P$ be a maximal $t$-ideal of $D$ which is distinct from all $P_{i}, i=1, \ldots, k$. If $P \notin\left\{M_{1}, \ldots, M_{n}\right\}$, then $a \notin P$, and hence (a) $D_{P}=D_{P}$. Suppose that $P \in\left\{M_{1}, \ldots, M_{n}\right\}$, say $P=M_{j}$ for some $1 \leq j \leq n$. Then, for some $b_{j} \in\left(\left\{a_{\alpha}^{m}\right\}\right) \backslash M_{j}$, we have $\left(b_{j}\right) D_{M_{j}}=D_{M_{j}}$. Let $I$ be the ideal of $D$ generated by $a, a_{1}^{\hat{m}_{1}}, \ldots, a_{k}^{\hat{m}_{k}}$ and the $b_{j}$ 's (if needed). Then $I$ is contained in $\left(\left\{a_{\alpha}^{m}\right\}\right)$ and $I$ is a finitely generated ideal of $D$, because the number of $b_{j}$ 's cannot exceed $n$. Let $P$ be a maximal $t$-ideal such that $P \neq P_{i}$ for all $1 \leq i \leq k$. If $a \notin P$, then $I D_{P}=D_{P}=\left(\left\{a_{\alpha}^{m}\right\}\right) D_{P}$. If $a \in P$, then $P=M_{j}$ for some $1 \leq j \leq n$; so $I D_{P}=D_{P}=\left(\left\{a_{\alpha}^{m}\right\}\right) D_{P}$ by the choice of a suitable $b_{j}$. Note that $I D_{P_{i}}=\left(\left\{a_{\alpha}^{m}\right\}\right) D_{P_{i}}$ for each $1 \leq i \leq k$. Thus $I D_{M}=\left(\left\{a_{\alpha}^{m}\right\}\right) D_{M}$ for all maximal $t$-ideals $M$ of $D$. It follows from [Kang 1989b, Proposition 2.8(3)] that $\left(\left\{a_{\alpha}^{m}\right\}\right)_{t}=I_{t}$, i.e., $\left(\left\{a_{\alpha}^{m}\right\}\right)_{t}$ is a finite type $t$-ideal of $D$. Hence $\left(\left\{a_{\alpha}^{m}\right\}\right)$ is $t$-invertible [Kang 1989b, Corollary 2.7], and thus $D$ is an AK-domain.

Recall that $D$ is a weakly Krull domain if $D=\bigcap_{P \in X^{1}(D)} D_{P}$ and this intersection has finite character. Note that if $t-\operatorname{dim}(D)=1$, then $X^{1}(D)=t-\operatorname{Max}(D)$. Thus, by Theorem 2.2, we have

Corollary 2.3. An AK-domain with $t$-dimension one is a weakly Krull domain.

However, the converse of Corollary 2.3 does not hold, and we provide such an example.

Example 2.4. Let $\mathbb{Q}($ resp., $\mathbb{R})$ be the field of rational (resp., real) numbers, $X$ be an indeterminate over $\mathbb{R}$, and $D=\mathbb{Q}+X \mathbb{R}[X]$.

(1) Note that $\mathbb{R}[X]$ is a Krull domain, and hence a weakly Krull domain. Thus $D$ is a weakly Krull domain [Anderson et al. 2006, Theorem 3.4], whence $t-\operatorname{dim}(D)=1$ [Anderson et al. 1992, Lemma 2.1].

(2) Let $F=\{a \in \mathbb{R} \mid a$ is integral over $\mathbb{Q}\}$. Clearly, $F$ is an integral domain. Let $0 \neq a \in F$. Then $a^{n}+q_{n-1} a^{n-1}+\cdots+q_{0}=0$ for some integer $n \geq 1$ and $q_{0}, \ldots, q_{n-1} \in \mathbb{Q}$ with $q_{0} \neq 0$; so

$$
\left(\frac{1}{a}\right)^{n}+\frac{q_{1}}{q_{0}}\left(\frac{1}{a}\right)^{n-1}+\cdots+\frac{1}{q_{0}}=0 .
$$

Hence $\frac{1}{a}$ is integral over $\mathbb{Q}$; so $F$ is a field. Also, it is easy to see that $\bar{D}=F+X \mathbb{R}[X]$, because $\mathbb{R}[X]$ is integrally closed and the quotient field of $D$ contains $\mathbb{R}[X]$. It is well known that $e \in \mathbb{R} \backslash F$, where $e=\lim _{n \rightarrow \infty}\left(1+\frac{1}{n}\right)^{n}$ is the base of the natural 
logarithm. Hence $\bar{D}$ is not a PvMD [Anderson and El Abidine 2001, Lemma 2.1]; so $D$ is not an APvMD [Li 2012, Theorem 3.6]. Thus $D$ is not an AK-domain.

Let $D \subseteq E$ be an extension of integral domains. Following [Dobbs et al. 1989], we say that $E$ is $t$-linked over $D$ if $I^{-1}=D$ for $I$ a nonzero finitely generated ideal of $D$ implies $(I E)^{-1}=E$; equivalently, if $M$ is a maximal $t$-ideal of $E$ with $M \cap D \neq(0)$, then $(M \cap D)_{t} \subsetneq D$ [Anderson et al. 1993, Proposition 2.1]. Anderson and Zafrullah introduced the concept of " $t$-linked under" which is the opposite notion of " $t$-linked over" [Anderson and Zafrullah 1991]. They defined that $D$ is $t$-linked under $E$ if for each nonzero finitely generated ideal $I$ of $D,(I E)^{-1}=E$ implies $I^{-1}=D$. Clearly, $D$ is $t$-linked under $E$ if and only if $(J E)_{t}=E$ implies $J_{t}=D$ for all nonzero ideals $J$ of $D$.

In [Anderson and Zafrullah 1991, Theorem 4.11], the authors showed that if $D \subseteq E$ is a bounded extension with $E \subseteq \bar{D}$, then $D$ is an API-domain (resp., AD-domain) if and only if $E$ is an API-domain (resp., AD-domain). Now, we give the AK-domain version of this result.

Theorem 2.5. Let $D \subseteq E$ be a bounded root extension with $E \subseteq \bar{D}$. Assume that $E$ is $t$-linked over D. Then $D$ is an AK-domain if and only if $E$ is an AK-domain and $D$ is $t$-linked under $E$.

Proof. $(\Rightarrow)$ Let $\left\{a_{\alpha}\right\}$ be a nonempty subset of $E \backslash\{0\}$. Since $D \subseteq E$ is a bounded root extension, there exists a positive integer $n$ such that $z^{n} \in D$ for all $z \in E$; so $\left\{a_{\alpha}^{n}\right\} \subseteq D$. Since $D$ is an AK-domain, there exists a positive integer $m=m\left(\left\{a_{\alpha}^{n}\right\}\right)$ such that $\left(\left\{a_{\alpha}^{n m}\right\}\right)$ is $t$-invertible, and hence $\left(\left(\left\{a_{\alpha}^{n m}\right\}\right) J\right)_{t}=D$ for some $v$-finite type ideal $J$ of $D$. Since $E$ is a $t$-linked overring of $D,\left(\left(\left(\left\{a_{\alpha}^{n m}\right\}\right) J\right) E\right)_{t}=E$. Thus $E$ is an AK-domain.

Let $I$ be a finitely generated ideal of $D$ such that $(I E)^{-1}=E$. Suppose to the contrary that $I_{v} \subsetneq D$. Then there exists a maximal $t$-ideal $M$ of $D$ such that $I_{t} \subseteq M$. Since $E \subseteq \bar{D}, M=M_{0} \cap D$ for some prime ideal $M_{0}$ of $E$ [Kaplansky 1994, Theorem 44]. Note that $D$ is a UMT-domain [Li 2012, Theorem 3.8]. Hence $M_{0}$ is a $t$-ideal of $E$ [Fontana et al. 1998, Proposition 1.4]. Therefore we have

$$
E=(I E)_{t} \subseteq(M E)_{t} \subseteq\left(M_{0}\right)_{t}=M_{0},
$$

which is impossible. Thus $I_{v}=D$ (or equivalently, $I^{-1}=D$ ), which says that $D$ is $t$-linked under $E$.

$(\Leftarrow)$ Assume that $E$ is an AK-domain and let $\left(\left\{b_{\beta}\right\}\right)$ be a nonzero ideal of $D$. Then there exists a positive integer $m=m\left(\left\{b_{\beta}\right\}\right)$ such that $\left(\left\{b_{\beta}^{m}\right\} E\right)$ is $t$-invertible, and hence $\left(\left(\left\{b_{\beta}^{m}\right\} E\right) J\right)_{t}=E$ for some $v$-finite ideal $J$ of $E$. Let $J=\left(\left\{j_{1}, \ldots, j_{l}\right\} E\right)_{t}$, where $0 \neq j_{1}, \ldots, j_{l} \in K$. Then we have

$$
\left(\left\{b_{\beta}^{m} j_{i}\right\} E\right)_{t}=\left(\left(\left\{b_{\beta}^{m}\right\} E\right)\left(\left\{j_{1}, \ldots, j_{l}\right\} E\right)\right)_{t}=\left(\left(\left\{b_{\beta}^{m}\right\} E\right) J\right)_{t}=E .
$$


Since $D \subseteq E$ is a bounded root extension, there is an integer $n \geq 1$ such that $b_{\beta}^{m n} j_{i}^{n}=\left(b_{\beta}^{m} j_{i}\right)^{n} \in D$ for all $b_{\beta}^{m} j_{i}$. Clearly, $\left(\left\{b_{\beta}^{m n} j_{i}^{n}\right\} E\right)_{t}=E$; so

$$
\left(\left(\left\{b_{\beta}^{m n}\right\} D\right)\left(\left\{j_{i}^{n}\right\} D\right)\right)_{t}=\left(\left\{b_{\beta}^{m n} j_{i}^{n}\right\} D\right)_{t}=D,
$$

because $D$ is $t$-linked under $E$. Thus $D$ is an AK-domain.

Corollary 2.6. If $D \subseteq \bar{D}$ is a bounded root extension, then the following statements are equivalent.

(1) $D$ is an AK-domain.

(2) $\bar{D}$ is a Krull domain and $D$ is $t$-linked under $\bar{D}$.

(3) (a) $t$-dim $(D)=1$,

(b) $D_{P}$ is an API-domain for each $P \in t-\operatorname{Max}(D)$, and

(c) $D=\bigcap_{P \in t-\operatorname{Max}(D)} D_{P}$ and this intersection has finite character.

Proof. (1) $\Leftrightarrow$ (2) Note that $\bar{D}$ is $t$-linked over $D$ [Anderson et al. 1993, Proposition 2.4], because $D \subseteq \bar{D}$ is a (bounded) root extension. Also, recall that an integrally closed AK-domain is a Krull domain. Thus the result follows from Theorem 2.5.

(2) $\Rightarrow$ (3) By Theorem 2.2, it suffices to show that $t-\operatorname{dim}(D)=1$. If $t-\operatorname{dim}(D) \geq 2$, then there are prime $t$-ideals $P_{1}, P_{2}$ of $D$ with $P_{1} \subsetneq P_{2}$. Let $Q_{1}, Q_{2}$ be prime ideals of $\bar{D}$ such that $Q_{i} \cap D=P_{i}$ for $i=1,2$. Note that $D$ is $t$-linked under $\bar{D}$; so $Q_{2}$ is a $t$-ideal, which means that $t-\operatorname{dim}(\bar{D}) \geq 2$, a contradiction. Thus $t-\operatorname{dim}(D)=1$.

(3) $\Rightarrow$ (1) Theorem 2.2.

Lemma 2.7. $D[\Gamma] \subsetneq D[X]$ is a (bounded) root extension if and only if $\operatorname{char}(D) \neq 0$.

Proof. If $D[\Gamma] \subsetneq D[X]$ is a root extension, then there is a positive integer $n$ such that $(1+X)^{n} \in D[\Gamma]$; so $n X \in D[\Gamma]$. Thus char $(D) \neq 0$. Conversely, assume that $\operatorname{char}(D)=p \neq 0$, and let $m$ be a positive integer such that $p^{m} \geq F(\Gamma)+1$. If $a+X g \in D[X]$, where $a \in D$ and $g \in D[X]$, then $(a+X g)^{p^{m}}=a^{p^{m}}+X^{p^{m}} g^{p^{m}}$ lies in $D[\Gamma]$. Thus $D[\Gamma] \subsetneq D[X]$ is a (bounded) root extension.

Let $t$-Spec $(A)$ be the set of prime $t$-ideals of an integral domain $A$. In [Chang et al. 2012, Theorem 1.5], we showed that the map $\varphi: t-\operatorname{Spec}(D[X]) \rightarrow t-\operatorname{Spec}(D[\Gamma])$, given by $Q \mapsto Q \cap D[\Gamma]$, is an order-preserving bijection. This shows that $D[X]$ is $t$-linked over $D[\Gamma]$, and at the same time $D[\Gamma]$ is $t$-linked under $D[X]$.

Corollary 2.8. If $D$ is a Krull domain, then $\operatorname{char}(D) \neq 0$ if and only if $D[\Gamma]$ is an AK-domain.

Proof. $(\Rightarrow)$ Note that $\overline{D[\Gamma]}=\bar{D}[X]=D[X]$; so $\overline{D[\Gamma]}$ is a Krull domain [Gilmer

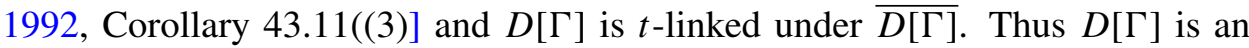
AK-domain by Corollary 2.6 and Lemma 2.7.

$(\Leftarrow)$ Since an AK-domain is an $\mathrm{AP} v \mathrm{MD}, D[\Gamma]$ is an $\mathrm{AP} v \mathrm{MD}$, and thus $\operatorname{char}(D) \neq$ 0 [Chang et al. 2012, Theorem 2.2]. 
Corollary 2.9. If $D[X] \subseteq \bar{D}[X]$ is a bounded root extension, then $D$ is an $A K$ domain if and only if $D[X]$ is an $A K$-domain.

Proof. This follows from Corollary 2.6, because $D$ is $t$-linked under $\bar{D}$ if and only if $D[X]$ is $t$-linked under $\bar{D}[X]$ [Anderson et al. 2004, Proposition 3.3].

Example 2.10. Let $F \subsetneq L$ be a pair of finite fields, and let $X, Y$ be indeterminates over $L$. Then $D=F+Y L \llbracket Y \rrbracket$ is an API-domain [Anderson et al. 1994, Example 3.8]; so $D$ is an AK-domain. Since $F[X] \subsetneq L[X]$ is not a root extension, $D[X] \subsetneq$ $\bar{D}[X]$ is not a (bounded) root extension [Anderson et al. 1994, Proposition 2.4]; so $D[X]$ is not an APvMD [Li 2012, Theorem 3.8]. Thus $D[X]$ is not an AK-domain. This shows that the assumption " $D[X] \subsetneq \bar{D}[X]$ is a bounded root extension" is essential in Corollary 2.9.

The next theorem characterizes when the numerical semigroup ring $D[\Gamma]$ is an AK-domain in terms of polynomial rings.

Theorem 2.11. $D[\Gamma]$ is an $A K$-domain if and only if $D[X]$ is an $A K$-domain and $\operatorname{char}(D) \neq 0$.

Proof. $(\Rightarrow)$ Assume that $D[\Gamma]$ is an AK-domain. Since an AK-domain is an $\operatorname{AP} v \mathrm{MD}, \operatorname{char}(D) \neq 0$ [Chang et al. 2012, Theorem 2.2]. Let $\left(\left\{f_{\alpha}\right\}\right)$ be a nonzero ideal of $D[X]$. Then there exists a positive integer $m$ such that $f_{\alpha}^{m} \in D[\Gamma]$ for all $f_{\alpha}$ by Lemma 2.7. Since $D[\Gamma]$ is an AK-domain, $\left(\left(\left\{f_{\alpha}^{r m}\right\}\right)\left(\left\{f_{\alpha}^{r m}\right\} D[\Gamma]\right)^{-1}\right)_{t}=D[\Gamma]$ for some integer $r=r\left(\left\{f_{\alpha}^{m}\right\}\right) \geq 1$. Note that $D[X]$ is $t$-linked over $D[\Gamma]$; so

$$
\left(\left(\left\{f_{\alpha}^{r m}\right\}\right)\left(\left\{f_{\alpha}^{r m}\right\} D[\Gamma]\right)^{-1} D[X]\right)_{t}=D[X] .
$$

Thus $D[X]$ is an AK-domain.

$(\Leftarrow)$ Let $\left(\left\{g_{\alpha}\right\}\right)$ be a nonzero ideal of $D[\Gamma]$. Since $D[X]$ is an AK-domain, there exists an integer $n=n\left(\left\{g_{\alpha}\right\}\right) \geq 1$ such that $\left(\left(\left\{g_{\alpha}^{n}\right\}\right) J D[X]\right)_{t}=D[X]$ for some $v$-finite type ideal $J$ of $D[X]$. Let $J=\left(\left(f_{1}, \ldots, f_{m}\right) D[X]\right)_{t}$, where $0 \neq f_{1}, \ldots, f_{m} \in K(X)$, the quotient field of $D[X]$. Then we have

$$
\left(\left(\left\{g_{\alpha}^{n} f_{i}\right\}\right) D[X]\right)_{t}=\left(\left(\left\{g_{\alpha}^{n}\right\}\right)\left(f_{1}, \ldots, f_{m}\right) D[X]\right)_{t}=D[X] .
$$

Since $\operatorname{char}(D) \neq 0, D[\Gamma] \subsetneq D[X]$ is a bounded root extension by Lemma 2.7; so there is an integer $k \geq 1$ such that $g_{\alpha}^{k n} f_{i}^{k} \in D[\Gamma]$ for all $g_{\alpha}^{n} f_{i}$. Also, it is obvious that $\left(\left(\left\{g_{\alpha}^{k n} f_{i}^{k}\right\}\right) D[X]\right)_{t}=D[X]$, because $\left(\left(\left\{g_{\alpha}^{n} f_{i}\right\}\right) D[X]\right)_{t}=D[X]$. Finally, since $D[\Gamma]$ is $t$-linked under $D[X]$, we obtain

$$
\left(\left(\left\{g_{\alpha}^{n k}\right\}\right)\left(\left\{f_{i}^{k}\right\}\right) D[\Gamma]\right)_{t}=\left(\left(\left\{g_{\alpha}^{n k} f_{i}^{k}\right\}\right) D[\Gamma]\right)_{t}=D[\Gamma] .
$$

Thus $D[\Gamma]$ is an AK-domain.

It is known that $D$ is a Krull domain if and only if each nonzero prime ideal of $D$ contains a $t$-invertible prime ideal [Kang 1989a, Theorem 3.6]. Also, we know 
that $D$ is a Krull domain if and only if $D$ is a Mori PvMD [Kang 1989a, Theorem 3.2]. So we have the following natural questions on AK-domains:

(1) Assume that each nonzero prime ideal of $D$ contains a nonzero prime ideal $P=\left(\left\{a_{\alpha}\right\}\right)$ such that $\left(\left\{a_{\alpha}^{n}\right\}\right)$ is $t$-invertible for some integer $n=n(P) \geq 1$. Is $D$ an AK-domain?

(2) Is a Mori APvMD an AK-domain?

We end this section by giving negative answers to these two questions.

Example 2.12. (1) Let $D$ be the integral domain as in Example 2.10. Then $D$ is a UMT-domain [Li 2012, Theorem 3.8], because an API-domain is an APvMD; so $t-\operatorname{dim}(D[X])=1$. Hence we have

$$
t-\operatorname{Max}(D[X])=\{Q \mid Q \text { is an upper to zero in } D[X]\} \cup\left\{P[X] \mid P \in X^{1}(D)\right\} \text {. }
$$

Note that if $Q \in t-\operatorname{Max}(D[X])$ with $Q \cap D=(0)$, then $Q$ is $t$-invertible. Next, if $P=\left(\left\{a_{\alpha}\right\}\right) \in X^{1}(D)$, then there exists a positive integer $n=n(P)$ such that $\left(\left\{a_{\alpha}^{n}\right\}\right)[X]$ is principal, and hence $t$-invertible.

(2) Let $p$ be a prime, $F=\bigcup_{n \geq 1} G F\left(p^{2^{n}}\right), L=F\left(G F\left(p^{3}\right)\right)$, and let $D=$ $F+X L \llbracket X \rrbracket$. Then $D$ is a one-dimensional quasilocal Noetherian AB-domain, but not an API-domain [Anderson et al. 1994, Example 3.6]. Thus $D$ is a Mori $\mathrm{AP} v \mathrm{MD}$, but not an AK-domain by Theorem 2.2.

\section{AUF-domains}

We say that $D$ is an $A U F$-domain if for every nonzero ideal $\left(\left\{a_{\alpha}\right\}\right)$ of $D$, there exists a positive integer $n=n\left(\left\{a_{\alpha}\right\}\right)$ such that $\left(\left\{a_{\alpha}^{n}\right\}\right)_{t}$ is principal. (Here, an AUF-domain stands for an almost unique factorization domain.)

We start this section with the AUF-domain analogue of the fact that $D$ is a UFD if and only if $D$ is a Krull domain and $C l(D)=0$.

Theorem 3.1. $D$ is an AUF-domain if and only if $D$ is an AK-domain and $C l(D)$ is torsion.

Proof. $(\Rightarrow)$ Let $I$ be a $t$-invertible $t$-ideal of $D$. Then $I=\left(a_{1}, \ldots, a_{k}\right)_{t}$ for some $a_{1}, \ldots, a_{k} \in D$. Since $D$ is an AUF-domain, $\left(I^{n}\right)_{t}=\left(a_{1}^{n}, \ldots, a_{k}^{n}\right)_{t}$ [Anderson and Zafrullah 1991, Lemma 3.3] is principal for some integer $n=n\left(a_{1}, \ldots, a_{k}\right) \geq 1$. Thus $C l(D)$ is torsion. Clearly, $D$ is an AK-domain.

$(\Leftarrow)$ Let $\left(\left\{b_{\alpha}\right\}\right)$ be a nonzero ideal of $D$. Since $D$ is an AK-domain, there exists a positive integer $n=n\left(\left\{b_{\alpha}\right\}\right)$ such that $\left(\left\{b_{\alpha}^{n}\right\}\right)_{t}$ is $t$-invertible. Also, since $C l(D)$ is torsion, $\left(\left\{b_{\alpha}^{n m}\right\}\right)_{t}=\left(\left(\left\{b_{\alpha}^{n}\right\}\right)^{m}\right)_{t}$ [Anderson and Zafrullah 1991, Lemma 3.3] is principal for some integer $m \geq 1$. Thus $D$ is an AUF-domain. 
Unlike the AK-domain case, an integrally closed AUF-domain need not be a UFD. For example, $\mathbb{Z}[\sqrt{-5}]$ is an integrally closed AUF-domain [Anderson and Zafrullah 1991, Theorem 4.17] which is not a UFD [Balcerzyk and Józefiak 1989, Example 3.4.1]. We give a characterization of integrally closed AUF-domains, which is also the analogue of the fact that $D$ is an integrally closed API-domain if and only if $D$ is a Dedekind domain with torsion class group [Anderson and Zafrullah 1991, Theorem 4.12].

Corollary 3.2. $D$ is an integrally closed AUF-domain if and only if $D$ is a Krull domain and $C l(D)$ is torsion.

Proof. Recall that an integrally closed AK-domain is a Krull domain. Thus this result comes directly from Theorem 3.1.

The next corollary explains the relationship between API-domains and AUFdomains.

Corollary 3.3. $D$ is an API-domain if and only if $D$ is an AUF-domain and each maximal ideal is a $t$-ideal.

Proof. $(\Rightarrow)$ Since an API-domain is an AD-domain, by Proposition 2.1, each maximal ideal of $D$ is a $t$-ideal. Clearly, $D$ is an AUF-domain.

$(\Leftarrow)$ Recall that $D$ is an API-domain if and only if $D$ is an AD-domain with torsion $t$-class group [Anderson and Zafrullah 1991, Lemma 4.4]. Thus the result is an immediate consequence of Proposition 2.1 and Theorem 3.1.

As in [Anderson et al. 1992], we say that $D$ is an almost weakly factorial domain (AWFD) if for each nonzero nonunit $d \in D$, there exists a positive integer $n=n(d)$ such that $d^{n}$ is a product of primary elements of $D$. It is known that $D$ is an AWFD if and only if $D$ is a weakly Krull domain with $C l(D)$ torsion [Anderson et al. 1992, Theorem 3.4]. Thus by Corollary 2.3 and Theorem 3.1, we have

Corollary 3.4. An AUF-domain with t-dimension one is an AWFD.

Remark 3.5. Let $D=\mathbb{Q}+X \mathbb{R}[X]$. Then $D$ is an AWFD [Anderson et al. 2006, Theorem 3.5], so $t$-dim $(D)=1$. Note that $D$ is not an AK-domain by Example 2.4. Hence by Theorem 3.1, $D$ is not an AUF-domain. Thus the converse of Corollary 3.4 is also not true.

The next two corollaries are analogues of Theorem 2.11 and Corollary 2.8, respectively.

Corollary 3.6. $D[\Gamma]$ is an AUF-domain if and only if $D[X]$ is an AUF-domain and $\operatorname{char}(D) \neq 0$.

Proof. Recall that $\operatorname{Pic}(K[\Gamma])$ is torsion if and only if $\operatorname{char}(D) \neq 0$ [Chang et al. 2012, Lemma 2.7]. Since $C l(D[\Gamma])=C l(D[X]) \oplus \operatorname{Pic}(K[\Gamma])$ [Anderson and 
Chang 2004, Theorem 5], $C l(D[\Gamma])$ is torsion if and only if $C l(D[X])$ is torsion and $\operatorname{char}(D) \neq 0$. Thus this equivalence follows from Theorems 2.11 and 3.1.

Corollary 3.7. If $D$ is a UFD, then $\operatorname{char}(D) \neq 0$ if and only if $D[\Gamma]$ is an $A U F$ domain.

Proof. The assertion follows from Corollary 3.6, because $D$ is a UFD if and only if $D[X]$ is a UFD [Zariski and Samuel 1975, Theorem 10, 117 , Chapter 1].

The AUF-domain version of Theorem 2.5 also carries over.

Theorem 3.8. Let $D \subseteq E$ be a bounded root extension with $E \subseteq \bar{D}$. Assume that $E$ is $t$-linked over $D$. Then $D$ is an AUF-domain if and only if $E$ is an AUF-domain and $D$ is $t$-linked under $E$.

Proof. $(\Rightarrow)$ Let $\left\{a_{\alpha}\right\}$ be a nonempty subset of $E \backslash\{0\}$. By the assumption, there exists a positive integer $n$ such that $\left\{a_{\alpha}^{n}\right\} \subseteq D$. Since $D$ is an AUF-domain, there exists a positive integer $m=m\left(\left\{a_{\alpha}^{n}\right\}\right)$ such that $\left(\left\{a_{\alpha}^{n m}\right\}\right)_{t}$ is principal, say $\left(\left\{a_{\alpha}^{n m}\right\}\right)_{t}=(d)$ for some $d \in D$. Hence $\left((1 / d)\left(\left\{a_{\alpha}^{n m}\right\}\right) D\right)_{t}=D$. Since $E$ is $t$-linked over $D$, $\left((1 / d)\left(\left\{a_{\alpha}^{n m}\right\}\right) E\right)_{t}=E$ [Anderson et al. 1993, Proposition 2.1]; so $\left(\left\{a_{\alpha}^{n m}\right\} E\right)_{t}=d E$. Thus $E$ is an AUF-domain. Since an AUF-domain is an AK-domain, by Theorem 2.5, $D$ is $t$-linked under $E$.

$(\Leftarrow)$ Assume that $E$ is an AUF-domain and let $\left(\left\{b_{\beta}\right\}\right)$ be a nonzero ideal of $D$. Then there exists a positive integer $m=m\left(\left\{b_{\beta}\right\}\right)$ such that $\left(\left\{b_{\beta}^{m}\right\} E\right)_{t}=x E$ for some $x \in E$; so $\left(\left\{b_{\beta}^{m} / x\right\} E\right)_{t}=E$. Since $D \subseteq E$ is a bounded root extension, there is an integer $n \geq 1$ such that $b_{\beta}^{m n} / x^{n}=\left(b_{\beta}^{m} / x\right)^{n} \in D$ for all $b_{\beta}^{m} / x$. Clearly, $\left(\left\{b_{\beta}^{m n} / x^{n}\right\} E\right)_{t}=E$, and since $D$ is $t$-linked under $E,\left(\left\{b_{\beta}^{m n} / x^{n}\right\} D\right)_{t}=D$. Therefore $\left(\left\{b_{\beta}^{m n}\right\}\right)_{t}=x^{n} D$, and thus $D$ is an AUF-domain.

By combining Theorem 3.8 with Corollary 3.2, we have

Corollary 3.9. If $D \subseteq \bar{D}$ is a bounded root extension, then $D$ is an AUF-domain if and only if $\bar{D}$ is a Krull domain, $C l(\bar{D})$ is torsion and $D$ is t-linked under $\bar{D}$.

Corollary 3.10. If $D[X] \subseteq \bar{D}[X]$ is a bounded root extension, then $D$ is an AUFdomain if and only if $D[X]$ is an AUF-domain.

Proof. Note that $C l(\bar{D}[X])=C l(\bar{D})$ [Gabelli 1987, Theorem 3.6] (or [El Baghdadi et al. 2002, Corollary 2.13]), $\bar{D}$ and $\bar{D}[X]$ are Krull domains simultaneously [Gilmer 1992 , Corollary 43.11(3)], and $D$ is $t$-linked under $\bar{D}$ if and only if $D[X]$ is $t$-linked under $\bar{D}[X]$ [Anderson et al. 2004, Proposition 3.3]. Thus the result is an immediate consequence of Corollary 3.9.

Remark 3.11. Example 2.10 also shows that the assumption " $D[X] \subsetneq \bar{D}[X]$ is a bounded root extension" is essential in Corollary 3.10. 
As an application of Corollary 3.10, we give an example of AUF-domains that is not an API-domain.

Corollary 3.12. Let $F \subsetneq L$ be a field extension, $0 \neq p=\operatorname{char}(F), X, Y$ be indeterminates over $L$ and let $D=F+Y L[Y]$ or $F+Y L \llbracket Y \rrbracket$. Assume that $L^{p^{n}} \subseteq F$ for some positive integer $n$. Then

(1) D is an API-domain.

(2) $D[X]$ is an AUF-domain that is not an API-domain.

Proof. (1) This appears in [Anderson et al. 1994, Corollary 2.2 and Theorem 3.5(3)].

(2) We do the case where $D=F+Y L[Y]$. Note that if $f \in D$, then $f^{p^{n}} \in$ $F[Y]$. Also, we note that $\bar{D}=L[Y]$. Let $f=a+g \in \bar{D}[X]$, where $a \in L$ and $g \in(X, Y) L[Y][X]$. Then $a^{p^{n}} \in F$; so $f^{p^{n}}$ belongs to $F+(X, Y) L[Y][X]$. Say $f^{p^{n}}=h_{0}+h_{1} X+\cdots+h_{m} X^{m}+g_{1} Y+\cdots+g_{k} Y^{k}$, where $h_{0} \in F, h_{i} \in L(1 \leq i \leq m)$ and $g_{j} \in L[X](1 \leq j \leq k)$. Since $L^{p^{n}} \subseteq F$ and $\operatorname{char}(F)=p$, we have

$$
\begin{aligned}
f^{p^{2 n}} & =\left(f^{p^{n}}\right)^{p^{n}} \\
& =h_{0}^{p^{n}}+h_{1}^{p^{n}} X^{p^{n}}+\cdots+h_{m}^{p^{n}} X^{p^{n}}+\sum_{j \geq 1}\left(g_{j} Y^{j}\right)^{p^{n}} \\
& \in F[X]+Y L[X][Y]=D[X] .
\end{aligned}
$$

Hence $D[X] \subseteq \bar{D}[X]$ is a bounded root extension, and thus $D[X]$ is an AUF-domain by Corollary 3.10 and (1). For the sake of contradiction, assume that $D[X]$ is an API-domain. Then $\overline{D[X]}=\bar{D}[X]$ is a Prüfer domain [Anderson and Zafrullah 1991, Corollary 4.8]. Therefore $\bar{D}$, and hence $D$ is a field [Gilmer 1992, Exercise 15 , Section 22], a contradiction. Thus $D[X]$ is not an API-domain.

A similar proof holds for the case where $D=F+Y L \llbracket Y \rrbracket$. (Note that if $D=F+Y L \llbracket Y \rrbracket$, then $\bar{D}=L \llbracket Y \rrbracket$.)

Example 3.13. (1) Let $F$ be a field with $\operatorname{char}(F)=p>0, X$ be an indeterminate over $F$ and $n$ be a positive integer. Then $F\left(X^{p^{n}}\right) \subsetneq F(X)$ is a field extension such that $F(X)^{p^{n}} \subseteq F\left(X^{p^{n}}\right)$.

(2) Let $D$ be an integral domain as in Corollary 3.12. Note that $\operatorname{char}(D) \neq 0$ and $D[X]$ is an AUF-domain. Hence by Corollary 3.6, $D[\Gamma]$ is a nonintegrally closed AUF-domain. This gives another example of an AUF-domain that is not a UFD.

Recall that a nonzero nonunit $p \in D$ is a prime block if for all $x, y \in D$ with $(x, p)_{v} \subsetneq D$ and $(y, p)_{v} \subsetneq D$, there exist an integer $n \geq 1$ and an element $d \in D$ such that $\left(x^{n}, y^{n}\right) \subseteq d D$ with $\left(x^{n} / d, p\right)_{v}=D$ or $\left(y^{n} / d, p\right)_{v}=D$. Following [Zafrullah 1985, Definition 1.10 and Remark 1.11], we say that $D$ is an almost unique factorization domain (AUFD) if for every nonzero nonunit $d \in D$, there is a positive integer $n=n(d)$ such that $d^{n}$ is expressible as a product of finitely many mutually $v$-coprime prime blocks; equivalently, every nonzero nonunit of $D$ 
is expressible as a product of finitely many prime blocks. (This explains why we use the name "AUF-domain" instead of AUFD.) It is obvious that each nonzero nonunit of a valuation domain $V$ is a prime block. Hence a valuation domain is an AUFD, but if $V$ has Krull dimension at least 2 or $V$ is a rank one nondiscrete valuation domain, then $V$ is not an AUF-domain since an integrally closed AUF-domain is a Krull domain by Corollary 3.2. This shows that the converse of the next theorem does not hold.

\section{Theorem 3.14. An AUF-domain with t-dimension one is an AUFD.}

Proof. Let $D$ be an AUF-domain of $t$-dimension one. If $P=\left(\left\{a_{\alpha}\right\}\right) \in X^{1}(D)$, then there exists an integer $m \geq 1$ such that $\left(\left\{a_{\alpha}^{m}\right\}\right)_{t}=p D$ for some $p \in D$. We claim that $p$ is a prime block. For $x, y \in D$ with $(x, p)_{v} \subsetneq D$ and $(y, p)_{v} \subsetneq D$, there exists an integer $n \geq 1$ such that $\left(x^{n}, y^{n}\right) \subseteq\left(x^{n}, y^{n}\right)_{v}=d D$ for some $d \in$ $D$. So $\left(x^{n} / d, y^{n} / d\right)_{v}=D$. Suppose to the contrary that $\left(x^{n} / d, p\right)_{v} \subsetneq D$ and $\left(y^{n} / d, p\right)_{v} \subsetneq D$. Since $t-\operatorname{dim}(D)=1$ and $P=\sqrt{p D} \in X^{1}(D)$, we have that $\left(x^{n} / d, y^{n} / d\right)_{v} \subseteq P \subsetneq D$, a contradiction. Thus $\left(x^{n} / d, p\right)_{v}=D$ or $\left(y^{n} / d, p\right)_{v}=D$ which indicates that $p$ is a prime block. Again, since $t-\operatorname{dim}(D)=1$, each nonzero prime ideal of $D$ contains a prime block. Thus $D$ is an AUFD [Zafrullah 1985, Theorem 2.2].

We say that $D$ is an almost $\pi$-domain if for every nonzero ideal $\left(\left\{a_{\alpha}\right\}\right)$ of $D$, there exists a positive integer $n=n\left(\left\{a_{\alpha}\right\}\right)$ such that $\left(\left\{a_{\alpha}^{n}\right\}\right)_{t}$ is invertible. (To prevent the reader's confusion, we should point out that our almost $\pi$-domain is different from Anderson's almost $\pi$-domain. He called $D$ an almost $\pi$-domain if $D$ is a Krull domain with torsion local $t$-class group [Anderson 1978, page 202]. Therefore, Anderson's almost $\pi$-domain is a special case of our almost $\pi$-domain. (See Theorem 3.15 or Corollary 3.16.)) It is clear that API-domain $\Rightarrow$ AUF-domain $\Rightarrow$ almost $\pi$-domain $\Rightarrow$ AK-domain.

The next theorem is the almost $\pi$-domain analogue of the result that $D$ is a $\pi$ domain if and only if $D$ is a Krull domain with trivial local $t$-class group [Anderson 1978, Theorem 1].

Theorem 3.15. $D$ is an almost $\pi$-domain if and only if $D$ is an AK-domain and $G(D)$ is torsion.

Proof. $(\Rightarrow)$ Let $I$ be a $t$-invertible $t$-ideal of $D$. Then $I=\left(a_{1}, \ldots, a_{m}\right)_{t}$ for some $a_{1}, \ldots, a_{m} \in D$. Since $D$ is an almost $\pi$-domain, there exists an integer $n=n\left(a_{1}, \ldots, a_{m}\right) \geq 1$ such that $\left(I^{n}\right)_{t}=\left(a_{1}^{n}, \ldots, a_{m}^{n}\right)_{t}$.Anderson and Zafrullah 1991, Lemma 3.3] is invertible. Thus $G(D)$ is torsion. Clearly, $D$ is an AK-domain.

$(\Leftarrow)$ If $\left(\left\{a_{\alpha}\right\}\right)$ is a nonzero ideal of an AK-domain $D$, then there exists a positive integer $n=n\left(\left\{a_{\alpha}\right\}\right)$ such that $\left(\left\{a_{\alpha}^{n}\right\}\right)_{t}$ is $t$-invertible. Since $G(D)$ is torsion, there is an integer $m=m\left(\left(\left\{a_{\alpha}^{n}\right\}\right)_{t}\right) \geq 1$ such that $\left(\left\{a_{\alpha}^{n m}\right\}\right)_{t}=\left(\left(\left\{a_{\alpha}^{n}\right\}\right)^{m}\right)_{t}$ [Anderson and Zafrullah 1991, Lemma 3.3] is invertible. Thus $D$ is an almost $\pi$-domain. 
Our remaining corollaries are the almost $\pi$-domain analogues of Corollaries 3.2 and 3.7, respectively.

Corollary 3.16. $D$ is an integrally closed almost $\pi$-domain if and only if $D$ is a Krull domain and $G(D)$ is torsion.

Proof. Recall again that an integrally closed AK-domain is a Krull domain. Thus the result comes directly from Theorem 3.15.

Example 3.17. Let $X, Y$ be analytic indeterminates over $\mathbb{Z}[\sqrt{-5}]$ and consider $D=\mathbb{Z}[\sqrt{-5}]\left[X^{2}, X Y, Y^{2}\right]$. Then $D$ is a Krull domain with $\operatorname{Pic}(D)=\mathbb{Z} / 2 \mathbb{Z} \subsetneq$ $\mathbb{Z} / 2 \mathbb{Z} \oplus \mathbb{Z} / 2 \mathbb{Z}=C l(D)$ [Anderson and Ryckaert 1988, Example 4.7(1)]; so $G(D)$ is torsion. Hence by Corollary 3.16, $D$ is an integrally closed almost $\pi$-domain. Note that $D$ is not a $\pi$-domain. Thus, an integrally closed almost $\pi$-domain need not be a $\pi$-domain.

Corollary 3.18. If $D$ is a $\pi$-domain, then $\operatorname{char}(D) \neq 0$ if and only if $D[\Gamma]$ is an almost $\pi$-domain.

Proof. Recall that a $\pi$-domain $D$ is a Krull domain with $C l(D)=\operatorname{Pic}(D)$. Therefore

$$
\begin{aligned}
C l(D[\Gamma]) & =C l(D[X]) \oplus \operatorname{Pic}(K[\Gamma]) \\
& =C l(D) \oplus \operatorname{Pic}(K[\Gamma]) \\
& \supseteq \operatorname{Pic}(D[\Gamma]) \\
& \supseteq C l(D) \oplus\{0\}
\end{aligned}
$$

where the first equality comes from [Anderson and Chang 2004, Theorem 5] and the second equality follows from [Gabelli 1987, Theorem 3.6] (or [El Baghdadi et al. 2002 , Corollary 2.13]). We also note that $\operatorname{char}(D) \neq 0$ if and only if $\operatorname{Pic}(K[\Gamma])$ is torsion [Chang et al. 2012, Lemma 2.7]. Hence if $D$ is a $\pi$-domain with nonzero characteristic, then $G(D[\Gamma])$ is torsion. Thus the result is an immediate consequence of Corollary 2.8 and Theorem 3.15.

It is worth remarking at this point that the assumption "bounded root extension" has a significant role in proving many results in our paper (for example, Theorems 2.5 and 3.8). In [Anderson and Zafrullah 1991], Anderson and Zafrullah also utilized this hypothesis to show some theorems about AB-domains, AP-domains, API-domains and AD-domains. Unfortunately, we are unable to prove Corollary 2.6 without this assumption, i.e., we do not know whether the $t$-dimension of an AKdomain is generally 1 . (Note that the $t$-dimension of a Krull domain is always 1.) We are closing this article with a couple of questions.

Question 3.19. Let $D$ be an AK-domain or AUF-domain.

(1) What is the $t$-dimension of $D$ ? Is it true that $t-\operatorname{dim}(D)=1$ ?

(2) Is $\bar{D}$ necessarily a Krull domain? 


\section{Appendix}

In this appendix, we give three diagrams of various integral domains related to (almost) factorization theory in order to help the readers better understand the correlation between the theory of almost factoriality and factorization theory. Each of the corresponding vertices in Figures 1 and 2 represent the correlation of integral domains in the relationship of the theory of almost factoriality and factorization theory. (For example, an AK-domain in the theory of almost factoriality is the corresponding notion to Krull domains in factorization theory.) In Figure 3, each vertex represents the integrally closed domain version of corresponding vertex in Figure 1. (For instance, an integrally closed API-domain is a Dedekind domain with torsion class group.) We also cite some well-known results about these domains.

For more on integral domains and almost factoriality see [Anderson et al. 1994; Anderson and Zafrullah 1991; Lewin 1997; Li 2012; Zafrullah 1985]. For more on integral domains in factorization theory, see [Anderson and Anderson 1980; Gilmer 1992; Kaplansky 1994; Mott and Zafrullah 1981].

Remark 1. Let $D$ be one of domains in Figure 1.

(1) In general, $D$ is not integrally closed.

(2) The arrows always hold, but none of the converse is true.

(3) Each implication of type $\leftarrow$ holds (for example, an AK-domain is an ADdomain) if and only if $\operatorname{Max}(D)=t-\operatorname{Max}(D)$.

(4) Each implication of type $\nearrow$ holds (for example, an AK-domain is an AUFdomain) if and only if $\mathrm{Cl}(D)$ is torsion.

(5) Each implication of type $\rightarrow$ holds (for example, an AK-domain is an almost $\pi$-domain) if and only if $G(D)$ is torsion.

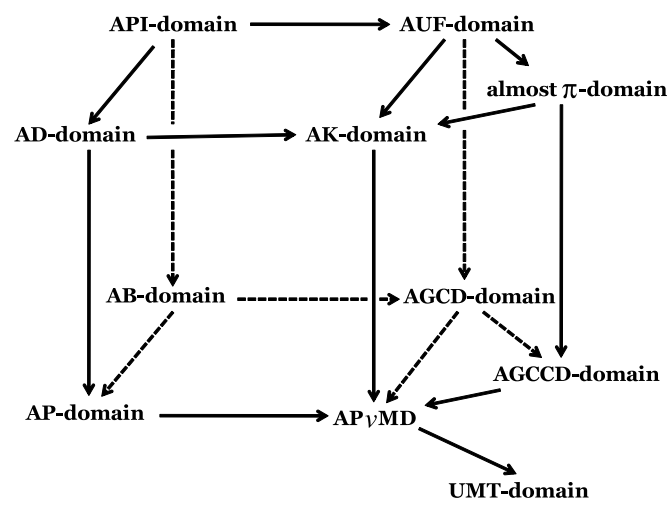

Figure 1. Nonintegrally closed domains in the theory of almost factoriality. 


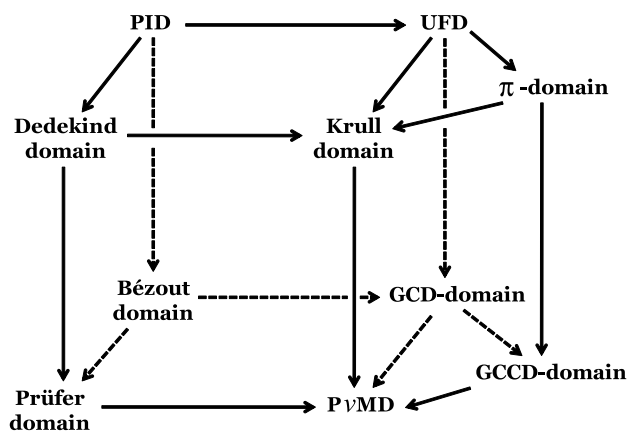

Figure 2. Integrally closed domains in factorization theory.

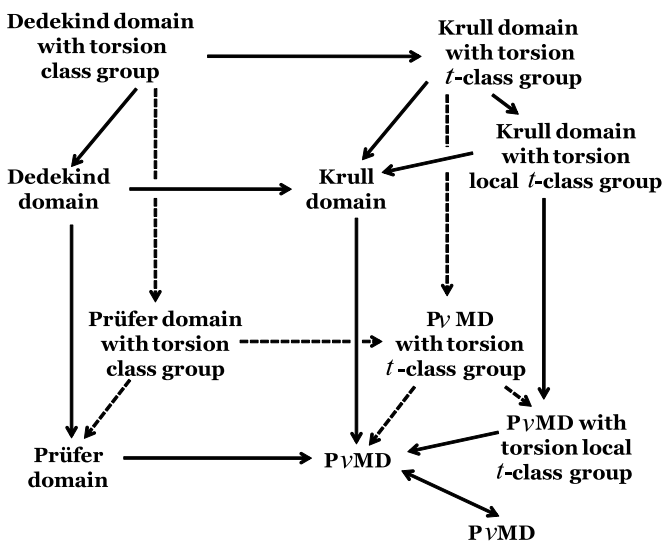

Figure 3. Integrally closed domain version of domains in the theory of almost factoriality.

Remark 2. Let $D$ denote one of domains in Figure 2.

(1) $D$ is integrally closed.

(2) The arrows always hold, but none of the converse holds.

(3) Each implication of type $\leftarrow$ holds (for instance, a Krull domain is a Dedekind domain) if and only if $\operatorname{Max}(D)=t-\operatorname{Max}(D)$.

(4) Each implication of type ${ }^{\nearrow}$ holds (for instance, a Krull domain is a UFD) if and only if $C l(D)=0$.

(5) Each implication of type $\rightarrow$ holds (for instance, a Krull domain is a $\pi$-domain) if and only if $G(D)=0$.

\section{Acknowledgements}

We would like to thank the referee for several valuable comments and suggestions, which resulted in an improved version of the paper. 


\section{References}

[Anderson 1978] D. D. Anderson, “ $\pi$-domains, overrings, and divisorial ideals”, Glasgow Math. J. 19:2 (1978), 199-203. MR 58 \#5647 Zbl 0379.13011

[Anderson and Anderson 1980] D. D. Anderson and D. F. Anderson, "Generalized GCD domains", Comment. Math. Univ. St. Paul. 28:2 (1980), 215-221. MR 81j:13017 Zbl 0434.13001

[Anderson and Chang 2004] D. F. Anderson and G. W. Chang, "The class group of $D[\Gamma]$ for $D$ an integral domain and $\Gamma$ a numerical semigroup", Comm. Algebra 32:2 (2004), 787-792. MR 2005g:13021 Zbl 1092.13017

[Anderson and El Abidine 2001] D. F. Anderson and D. N. El Abidine, "The $A+X B[X]$ and $A+X B \llbracket X \rrbracket$ constructions from GCD-domains”, J. Pure Appl. Algebra 159:1 (2001), 15-24. MR 2002f:13037 Zbl 1004.13004

[Anderson and Ryckaert 1988] D. F. Anderson and A. Ryckaert, "The class group of $D+M$ ", $J$. Pure Appl. Algebra 52:3 (1988), 199-212. MR 89h:13008 Zbl 0668.13013

[Anderson and Zafrullah 1991] D. D. Anderson and M. Zafrullah, "Almost Bézout domains", J. Algebra 142:2 (1991), 285-309. MR 92g:13026 Zbl 0749.13013

[Anderson et al. 1992] D. D. Anderson, J. L. Mott, and M. Zafrullah, "Finite character representations for integral domains", Boll. Un. Mat. Ital. B (7) 6:3 (1992), 613-630. MR 93k:13001 Zbl 0773.13004

[Anderson et al. 1993] D. D. Anderson, E. G. Houston, and M. Zafrullah, " $t$-linked extensions, the $t$-class group, and Nagata's theorem”, J. Pure Appl. Algebra 86:2 (1993), 109-124. MR 94e:13036 Zbl 0777.13002

[Anderson et al. 1994] D. D. Anderson, K. R. Knopp, and R. L. Lewin, "Almost Bézout domains, II", J. Algebra 167:3 (1994), 547-556. MR 95f:13026 Zbl 0821.13006

[Anderson et al. 2004] D. D. Anderson, T. Dumitrescu, and M. Zafrullah, "Almost splitting sets and AGCD domains”, Comm. Algebra 32:1 (2004), 147-158. MR 2005d:13002 Zbl 1063.13002

[Anderson et al. 2006] D. F. Anderson, G. W. Chang, and J. Park, "Weakly Krull and related domains of the form $D+M, A+X B[X]$ and $A+X^{2} B[X]$ ", Rocky Mountain J. Math. 36:1 (2006), 1-22. MR 2007h:13027 Zbl 1133.13022

[Balcerzyk and Józefiak 1989] S. Balcerzyk and T. Józefiak, Commutative Noetherian and Krull rings, Ellis Horwood, Chichester, 1989. MR 92f:13001 Zbl 0685.13001

[Chang et al. 2012] G. W. Chang, H. Kim, and J. W. Lim, "Numerical semigroup rings and almost Prüfer v-multiplication domains", Comm. Algebra 40:7 (2012), 2385-2399.

[Dobbs et al. 1989] D. E. Dobbs, E. G. Houston, T. G. Lucas, and M. Zafrullah, " $t$-linked overrings and Prüfer $v$-multiplication domains”, Comm. Algebra 17:11 (1989), 2835-2852. MR 90j:13016 Zbl 0691.13015

[El Baghdadi et al. 2002] S. El Baghdadi, L. Izelgue, and S. Kabbaj, "On the class group of a graded domain”, J. Pure Appl. Algebra 171:2-3 (2002), 171-184. MR 2003d:13012 Zbl 1058.13006

[Fontana et al. 1998] M. Fontana, S. Gabelli, and E. Houston, "UMT-domains and domains with Prüfer integral closure”, Comm. Algebra 26:4 (1998), 1017-1039. MR 99d:13024 Zbl 0930.13014

[Gabelli 1987] S. Gabelli, "On divisorial ideals in polynomial rings over Mori domains", Comm. Algebra 15:11 (1987), 2349-2370. MR 88m:13006 Zbl 0642.13017

[Gilmer 1992] R. Gilmer, Multiplicative ideal theory, Queen's Papers in Pure and Applied Mathematics 90, Queen's University, Kingston, ON, 1992. MR 93j:13001 Zbl 0804.13001

[Houston and Zafrullah 1989] E. Houston and M. Zafrullah, "On $t$-invertibility, II", Comm. Algebra 17:8 (1989), 1955-1969. MR 90i:13019 Zbl 0717.13002 
[Kang 1989a] B. G. Kang, "On the converse of a well-known fact about Krull domains", J. Algebra 124:2 (1989), 284-299. MR 90g:13033 Zbl 0694.13011

[Kang 1989b] B. G. Kang, "Prüfer $v$-multiplication domains and the ring $R[X]_{N_{v}}$ ", J. Algebra 123:1 (1989), 151-170. MR 90e:13017 Zbl 0668.13002

[Kaplansky 1994] I. Kaplansky, Commutative rings, Polygonal, Washington, NJ, 1994.

[Lewin 1997] R. L. Lewin, "Almost generalized GCD-domains", pp. 371-382 in Factorization in integral domains (Iowa City, IA, 1996), edited by D. D. Anderson, Lecture Notes in Pure and Appl. Math. 189, Marcel Dekker, New York, 1997. MR 98i:13043 Zbl 0909.13009

[Li 2012] Q. Li, “On almost Prüfer v-multiplication domains”, Algebra Colloq. 19:3 (2012), 493-500.

[Mott and Zafrullah 1981] J. L. Mott and M. Zafrullah, "On Prüfer v-multiplication domains", Manuscripta Math. 35:1-2 (1981), 1-26. MR 83d:13026 Zbl 0477.13007

[Pirtle 1968] E. M. Pirtle, Jr., "Integral domains which are almost-Krull", J. Sci. Hiroshima Univ. Ser. A-I Math. 32 (1968), 441-447. MR 39 \#5538 Zbl 0181.04903

[Sahandi 2010] P. Sahandi, "Minimal prime ideals and semistar operations", Rocky Mountain J. Math. 40:3 (2010), 1039-1049. MR 2011h:13003 Zbl 1200.13008

[Storch 1967] U. Storch, Fastfaktorielle Ringe, Schr. Math. Inst. Univ. Münster 36, Universität Münster, Münster, 1967. MR 35 \#5437 Zbl 0168.28901

[Zafrullah 1985] M. Zafrullah, "A general theory of almost factoriality", Manuscripta Math. 51:1-3 (1985), 29-62. MR 86m:13023 Zbl 0587.13010

[Zariski and Samuel 1975] O. Zariski and P. Samuel, Commutative algebra, vol. 1, Graduate Texts in Mathematics 28, Springer, New York, 1975. MR 52 \#5641 Zbl 0313.13001

Received January 14, 2012. Revised March 15, 2012.

GYU WHAN CHANG

DEPARTMENT OF MATHEMATICS

UNIVERSITY OF INCHEON

INCHEON 406-772

SOUTH KOREA

whan@incheon.ac.kr

HWANKOO KIM

DEPARTMENT OF INFORMATION SECURITY

HOSEO UNIVERSITY

ASAN 336-795

SOUTH KOREA

hkkim@hoseo.edu

JUNG WOOK LIM

DEPARTMENT OF MATHEMATICS

SOGANG UNIVERSITY

SEOUL 121-742

SOUTH KOREA

lovemath@postech.ac.kr

jwlim@sogang.ac.kr 


\title{
PACIFIC JOURNAL OF MATHEMATICS
}

\author{
http://pacificmath.org \\ Founded in 1951 by \\ E. F. Beckenbach (1906-1982) and F. Wolf (1904-1989)
}

\section{EDITORS}

V. S. Varadarajan (Managing Editor)

Department of Mathematics

University of California

Los Angeles, CA 90095-1555

pacific@math.ucla.edu

Vyjayanthi Chari

Department of Mathematics

University of California

Riverside, CA 92521-0135

chari@math.ucr.edu

\section{Robert Finn}

Department of Mathematics Stanford University

Stanford, CA 94305-2125

finn@math.stanford.edu

Kefeng Liu

Department of Mathematics

University of California

Los Angeles, CA 90095-1555

liu@math.ucla.edu
Darren Long

Department of Mathematics

University of California

Santa Barbara, CA 93106-3080

long@math.ucsb.edu

Jiang-Hua Lu

Department of Mathematics

The University of Hong Kong

Pokfulam Rd., Hong Kong jhlu@maths.hku.hk

Alexander Merkurjev

Department of Mathematics

University of California

Los Angeles, CA 90095-1555

merkurev@math.ucla.edu
Sorin Popa

Department of Mathematics University of California

Los Angeles, CA 90095-1555 popa@math.ucla.edu

Jie Qing

Department of Mathematics

University of California

Santa Cruz, CA 95064

qing@cats.ucsc.edu

Jonathan Rogawski

Department of Mathematics

University of California

Los Angeles, CA 90095-1555

jonr@math.ucla.edu

\section{PRODUCTION}

pacific@math.berkeley.edu

\section{SUPPORTING INSTITUTIONS}

ACADEMIA SINICA, TAIPEI

CALIFORNIA INST. OF TECHNOLOGY INST. DE MATEMÁTICA PURA E APLICADA KEIO UNIVERSITY

MATH. SCIENCES RESEARCH INSTITUTE NEW MEXICO STATE UNIV.

OREGON STATE UNIV.

\author{
STANFORD UNIVERSITY \\ UNIV. OF BRITISH COLUMBIA \\ UNIV. OF CALIFORNIA, BERKELEY \\ UNIV. OF CALIFORNIA, DAVIS \\ UNIV. OF CALIFORNIA, LOS ANGELES \\ UNIV. OF CALIFORNIA, RIVERSIDE \\ UNIV. OF CALIFORNIA, SAN DIEGO \\ UNIV. OF CALIF., SANTA BARBARA
}

\author{
UNIV. OF CALIF., SANTA CRUZ \\ UNIV. OF MONTANA \\ UNIV. OF OREGON \\ UNIV. OF SOUTHERN CALIFORNIA \\ UNIV. OF UTAH \\ UNIV. OF WASHINGTON \\ WASHINGTON STATE UNIVERSITY
}

These supporting institutions contribute to the cost of publication of this Journal, but they are not owners or publishers and have no responsibility for its contents or policies.

See inside back cover or pacificmath.org for submission instructions.

The subscription price for 2012 is US \$420/year for the electronic version, and \$485/year for print and electronic.

Subscriptions, requests for back issues from the last three years and changes of subscribers address should be sent to Pacific Journal of Mathematics, P.O. Box 4163, Berkeley, CA 94704-0163, U.S.A. Prior back issues are obtainable from Periodicals Service Company, 11 Main Street, Germantown, NY 12526-5635. The Pacific Journal of Mathematics is indexed by Mathematical Reviews, Zentralblatt MATH, PASCAL CNRS Index, Referativnyi Zhurnal, Current Mathematical Publications and the Science Citation Index.

The Pacific Journal of Mathematics (ISSN 0030-8730) at the University of California, c/o Department of Mathematics, 969 Evans Hall, Berkeley, CA 94720-3840, is published monthly except July and August. Periodical rate postage paid at Berkeley, CA 94704, and additional mailing offices. POSTMASTER: send address changes to Pacific Journal of Mathematics, P.O. Box 4163, Berkeley, CA 94704-0163.

PJM peer review and production are managed by EditFLOW ${ }^{\mathrm{TM}}$ from Mathematical Sciences Publishers.

PUBLISHED BY PACIFIC JOURNAL OF MATHEMATICS

at the University of California, Berkeley 94720-3840

A NON-PROFIT CORPORATION

Typeset in LATEX

Copyright $(02012$ by Pacific Journal of Mathematics 


\section{PACIFIC JOURNAL OF MATHEMATICS}

Volume $260 \quad$ No. $1 \quad$ November 2012

The decomposition of global conformal invariants: Some technical proofs II

SPYROS ALEXAKIS

On deformation quantizations of hypertoric varieties

GWYN BELLAMY and TOSHIRO KUWABARA

Almost factoriality of integral domains and Krull-like domains

GYU Whan CHANG, HWANKOO KIM and JUNG WOOK LIM

Singularities of free group character varieties

CARLOS FLORENTINO and SEAN LAWTON

Energy identity for the maps from a surface with tension field bounded 181 in $L^{p}$

LI JIAYU and ZHU XIANGRONG

Remarks on some isoperimetric properties of the $k-1$ flow

YU-CHU LIN and DONG-Ho TSAI

Demystifying a divisibility property of the Kostant partition function 215

KAROLA MÉSZÁros

Exceptional Lie algebras, SU(3), and Jordan pairs

PIERO TRUINI

Lower estimate of Milnor number and characterization of isolated homogeneous hypersurface singularities

Stephen S.-T. Yau and HuAiqing Zuo 